\title{
The role of LASS2 in regulating bladder cancer cell tumorigenicity in a nude mouse model
}

\author{
YUJIN CHEN $^{1,2^{*}}$, HAIFENG WANG $^{1 *}$, TAO XIONG $^{3 *}$, \\ RENCHAO ZOU ${ }^{1}$, ZHAORAN TANG $^{1}$ and JIANSONG WANG ${ }^{1}$
}

\begin{abstract}
${ }^{1}$ Department of Urology, The Second Affiliated Hospital of Kunming Medical University, Yunnan Institute of Urology, Kunming, Yunnan 650101; ${ }^{2}$ Department of Nephrology, Chuxiong People's Hospital, Chuxiong, Yunnan 675000; ${ }^{3}$ Department of Cadre Medical Care, The First Affiliated Hospital of Kunming Medical University, Kunming, Yunnan 650031, P.R. China
\end{abstract}

Received February 16, 2016; Accepted January 26, 2017

DOI: $10.3892 / 01.2017 .6880$

\begin{abstract}
Previous in vitro studies have demonstrated that LAG1 longevity assurance homolog 2 (LASS2) is a novel tumor suppressor gene that is significantly associated with the proliferation and invasion ability of tumor cells. However, the role LASS2 serves in regulating bladder cancer cell tumorigenicity and tumor growth in vivo has not yet been elucidated in animal or clinical studies. In the present study, LASS2 knockdown in human bladder cancer EJ-M3 cells significantly promoted the growth of xenografts in nude mice compared with the control group, while overexpression of LASS2 suppressed tumor growth; however, this was not statistically significant. Furthermore, LASS2 knockdown resulted in more apparent heteromorphism and a higher activity of matrix metalloproteinase (MMP)-2 and MMP-9 in xenograft tumors. The data from the present study demonstrated that LASS2 knockdown significantly promoted the tumorigenicity and growth of EJ-M3 xenograft tumors in nude mice, and that LASS2 overexpression has a tendency to inhibit the growth of xenografts, suggesting that it may be a potential therapeutic target for bladder cancer.
\end{abstract}

Correspondence to: Professor Jiansong Wang, Department of Urology, The Second Affiliated Hospital of Kunming Medical University, Yunnan Institute of Urology, 374 Dianmian Road, Kunming, Yunnan 650101, P.R. China

E-mail: jiansongwangkm@126.com

*Contributed equally

Abbreviations: LASS2, LAG1 longevity assurance homologue 2; GFP, green fluorescent protein; RT-qPCR, quantitative real-time polymerase chain reaction; H\&E, hematoxylin and eosin; MMPs, matrix metalloproteinases; HCC, hepatocellular carcinoma

Key words: bladder cancer cell, tumorigenicity, tumor growth, nude mice, LASS2

\section{Introduction}

Bladder cancer is one of the most common malignant tumors of the urinary system and is a major cause of mortality worldwide. An estimated 429,800 cases of bladder cancer are diagnosed every year and 165,100 mortalities occurred in 2012 worldwide as a result of the disease (1). In the United States of America, a total of 79,030 new bladder cancer cases and 16,870 bladder cancer-associated mortalities were projected to occur in $2017(2,3)$. Therefore, the identification of genes associated with bladder cancer and novel therapeutic targets is necessary.

LAG1 longevity assurance homolog 2 (LASS2), also known as tumor metastasis suppressor gene 1, was identified as a tumor suppressor gene (3). LASS2 has been correlated with the degree of invasion and recurrence of carcinomas of the prostate $(4-6)$, liver $(7)$, breast $(8,9)$ and bladder $(10)$. However, to the best of our knowledge, the precise role LASS2 serves in regulating bladder cancer cell tumorigenicity and growth in vivo has not yet been investigated in animal or clinical studies.

In previous studies, an implantation metastasis model of the highly invasive human bladder cancer EJ-M3 cell line was established in nude mice $(11,12)$. Another study identified that LASS2 expression was the highest in the EJ-M3 cell line compared with other human bladder carcinoma cell lines (BIU-87, T24 and EJ), and was significantly correlated with the proliferation, metastasis and invasion of human bladder cancer (13). In the present study, EJ-M3 cells were selected for a lentivirus-mediated LASS2 interference strategy and LASS2 overexpression, in order to investigate the effects of LASS2 expression on bladder cancer cell tumorigenicity and growth in vitro and in nude mice.

\section{Materials and methods}

Cell line and cell culture. The highly invasive human bladder cancer EJ-M3 cell line was established and preserved at the Department of Urology of the Second Affiliated Hospital of Kunming Medical University (Yunnan Institute of Urology, Kunming, China) (11). Cells were maintained in RPMI-1640 supplemented with $10 \%$ fetal bovine serum (Gibco; Thermo 
Fisher Scientific, Inc., Waltham, MA, USA), $100 \mathrm{U} / \mathrm{ml}$ penicillin and $100 \mu \mathrm{g} / \mathrm{ml}$ streptomycin at $37^{\circ} \mathrm{C}$ in a humidified atmosphere with $5 \% \mathrm{CO}_{2}$. All reagents were purchased from EMD Millipore (Billerica, MA, USA).

Construction of the lentiviral vector and cell transfection. EJ-M3 cells were transfected with a LASS2 shRNA plasmid (cat no. HSH008629-CU6) or a LASS2 overexpression plasmid (cat no. EX-T3019-M98-5) (both GeneCopoeia, Inc., Rockville, MD, USA). Briefly, the plasmids were amplified in DH5a Escherichia coli cells (Takara Biotechnology Co., Ltd., Dalian, China), purified using an E.Z.N.A. ${ }^{\circledR}$ Endo-Free Plasmid Mini Kit I (Omega Bio-Tek, Inc., Norcross, GA, USA), and transfected into $80 \%$ confluent EJ-M3 cells using Lipofectamine $^{\circledR} 2000$ (Invitrogen; Thermo Fisher Scientific, Inc., Waltham, MA, USA). A lentiviral vector expressing green fluorescent protein (GFP) alone was used as the control. At $72 \mathrm{~h}$ following transfection, LASS2 overexpression and LASS2 shRNA positive cells were selected using $1 \mu \mathrm{g} / \mathrm{ml}$ neomycin or puromycin, respectively, and the transfection efficiency was examined by fluorescence microscopy (Olympus BX53; Olympus Corporation, Tokyo, Japan).

Reverse transcription-quantitative polymerase chain reaction (RT-qPCR) analysis. Total RNA was extracted from cells using TRIzol reagent (Invitrogen; Thermo Fisher Scientific, Inc.), and reverse transcribed into cDNA using Quant Reverse Transcriptase (Tiangen Biotech Co., Ltd., Beijing, China) following the manufacturer's protocol. $\beta$-Actin was used as the internal control. RT-qPCR was performed in a $20 \mu \mathrm{l}$ reaction containing $1 \mu \mathrm{l}$ cDNA template, $1 \mu \mathrm{l}$ primer and $10 \mu \mathrm{l}$ FastStart Universal SYBR Green Master (ROX) (Roche Ltd., Mannheim, Germany), using an Applied Biosystems 7900HT Real-Time PCR System (Applied Biosystems; Thermo Fisher Scientific Ltd., Waltham, MA, USA). The primers sequences were as follows: $\beta$-actin forward, 5'-GAAGGTGAAGGTCGG AGTC-3'; and reverse, 5'-GAAGATGGTGATGGGATTTC-3'; LASS2 forward, 5'-TCTCCTGGTTTGCCA ATTACG-3'; and reverse, 5'-CCGGGCAGGGACCCTCATCA-3'. All the primers were synthesized by GeneCopoeia, Inc. The amplification program consisted of denaturation at $95^{\circ} \mathrm{C}$ for $1 \mathrm{~min}$, followed by 40 cycles of denaturation at $95^{\circ} \mathrm{C}$ for $15 \mathrm{sec}$ and annealing at $60^{\circ} \mathrm{C}$ for $30 \mathrm{sec}$, and then extension at $60^{\circ} \mathrm{C}$ for $30 \mathrm{sec}$. All experiments were repeated at least three times. The relative expression of LASS2 mRNA was calculated using the $2^{-\Delta \Delta \mathrm{Cq}}$ method (14).

Western blot analysis. Cell lysates were extracted using RIPA Lysis Buffer (Beyotime Institute of Biotechnology, Haimen, China). Total proteins were separated via SDS-PAGE on a $10 \%$ gel, transferred onto $0.45 \mu \mathrm{m}$ polyvinylidene difluoride membranes (EMD Millipore), and blocked with 5\% skimmed milk. Using $\beta$-actin as the internal control, the membranes were incubated with a mouse anti-LASS2 (cat. no. sc-390745; dilution, 1:500) or anti- $\beta$-actin monoclonal antibody (cat. no. sc-47778; dilution, 1:500) (Santa Cruz Biotechnology, Inc., Dallas, TX, USA) overnight at $4^{\circ} \mathrm{C}$, followed by incubation with a horseradish peroxidase-conjugated goat anti-mouse immunoglobulin G (cat. no. sc-2005; dilution, 1:2,500; Santa Cruz Biotechnology, Inc.) at room temperature for $1 \mathrm{~h}$.
Protein bands were visualized through enhanced chemiluminescence by using Immobilon Western Chemiluminescent HRP substrate (EMD Millipore Corporation, Billerica, MA, USA) and captured using a MicroChemi 4.2 system (DNR Bio-Imaging Systems Ltd., Jerusalem, Israel). Relative protein quantitative quantification was performed using ImageJ image analysis software (version 1.34; National Institutes of Health, Bethesda, MD, USA).

Establishment of a human bladder cancer xenograft model in nude mice. The animal experiments were approved by the Institutional Animal Care and Use Committee of Kunming Medical University (Kunming, China) (approval no. KYLL20140071), and were performed in compliance with all regulatory institutional guidelines for animal welfare (the National Institutes of Health Publications no. 80-23). Fifteen 6-week-old female nude mice (BALB/c-nu/nu) weighing 16-20 g, were purchased from Beijing HFK Bioscience Company (Beijing, China), and were randomly divided into the following 3 groups (5/group): A non-transfected EJ-M3 cell group [control (Con)]; a LASS2-overexpression EJ-M3 cell group (LASS2-over); and a LASS2-shRNA EJ-M3 cell group (LASS2-shRNA). All nude mice were housed in a sterile environment with a room temperature of $20-25^{\circ} \mathrm{C}$, a relative humidity of $30-70 \%$ and a $12 \mathrm{~h} \mathrm{light/dark} \mathrm{cycle.}$ All mice were given free access to food and water, and the bedding materials, drinking water, feeding cages and other items in contact with the animals were all autoclaved prior to use. Cell suspensions $\left(200 \mu 1,2 \times 10^{6} / \mathrm{ml}\right)$ of non-transfected, LASS2-over or LASS2-shRNA EJ-M3 cells were subcutaneously injected into the right side of the dorsal aspect of the nude mice using a 6-gauge needle. The tumor size and body weight of each mouse were measured once a week. The tumor volumes were calculated using the following formula: $\mathrm{V}=\pi / 6 \mathrm{x}$ largest diameter $\mathrm{x}$ smallest diameter ${ }^{2}(15)$, and tumor growth curves were plotted. The tumor inhibition rate (TIR) was determined using the formula: TIR $(\%)=[1-($ mean tumor volume of experimental group/mean tumor volume of control group)]x100. All mice were sacrificed 6 weeks following injection, and the tumors were resected. One part of each tumor was fixed in $4 \%$ formalin, dehydrated with gradient alcohol, cleared with xylene, embedded in paraffin, sectioned serially (4- $\mu$ m-thick sections), and then mounted on a slide for hematoxylin and eosin staining and immunohistochemical analysis. Another part of each tumor was stored at $-80^{\circ} \mathrm{C}$ for the detection of matrix metalloproteinase (MMP)-2/-9 activity. The slides were stained with hematoxylin and eosin (H\&E), and the properties of the xenograft tumors were assessed under an optical microscope (Eclipse E200; Nikon Corporation, Tokyo, Japan).

Immunohistochemical analysis. The slides were heated and subsequently put in xylene, washed with gradient alcohol for deparaffinization, and then rehydrated with distilled water. After that, antigen retrieval was performed in $1 \mathrm{mM}$ boiling EDTA buffer ( $\mathrm{pH} \mathrm{8.0;} \mathrm{EMD} \mathrm{Millipore)} \mathrm{for} 15 \mathrm{~min}$ at $92-100^{\circ} \mathrm{C}$. The slides were incubated with $3 \%$ hydrogen peroxide for $30 \mathrm{~min}$, washed, and blocked with $5 \%$ goat serum (EMD Millipore) for $60 \mathrm{~min}$ at room temperature. A mouse monoclonal anti-LASS2 antibody (cat. no. sc-390745; dilution, 
A

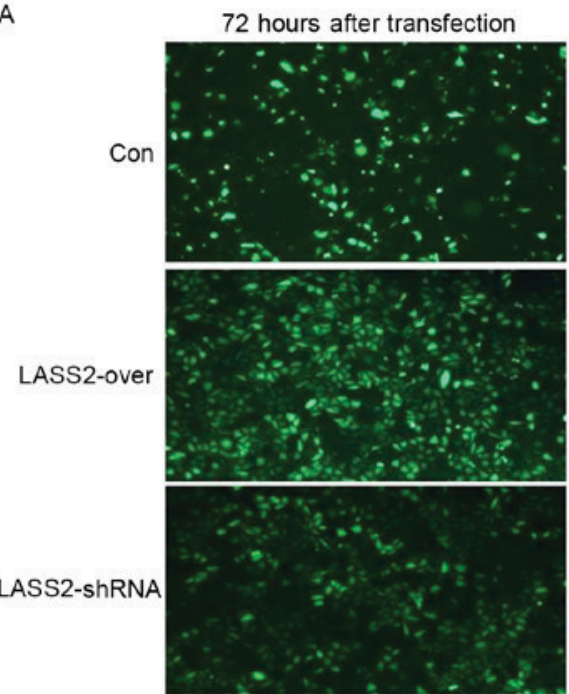

C

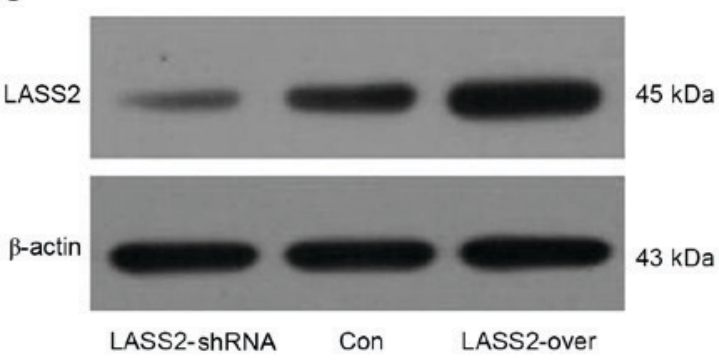

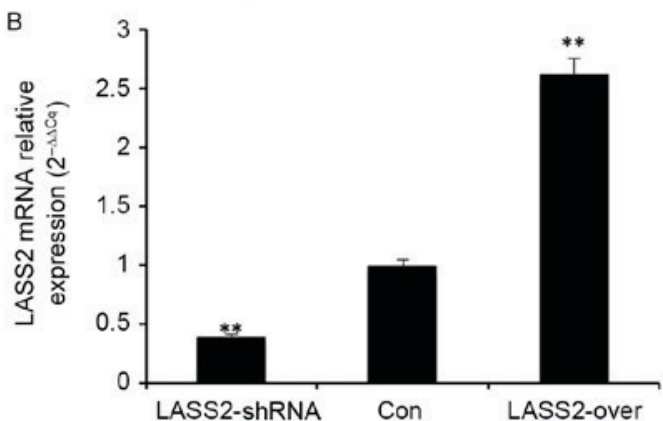

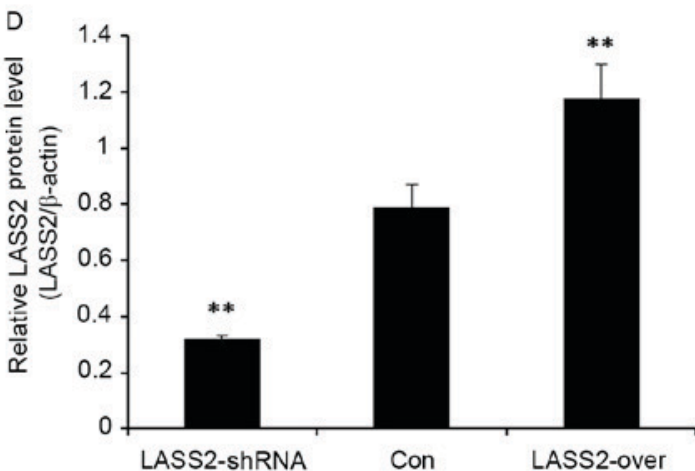

Figure 1. Expression of LASS2 in EJ-M3 cells following transfection. (A) EJ-M3 cells were transfected with a LASS2 overexpression plasmid or a LASS2 shRNA construct. Fluorescence images were obtained at $72 \mathrm{~h}$ following transfection (magnification, x100). (B) LASS2 relative mRNA expression levels were determined by RT-qPCR analysis following transfection. (C) Western blot analysis of LASS2 protein levels following transfection. $\beta$-Actin was used as the internal control. (D) LASS2 protein levels relative to $\beta$-actin were quantified using ImageJ software. Non-infected EJ-M3 cells were used as the Con group. ${ }^{*} \mathrm{P}<0.05,{ }^{* *} \mathrm{P}<0.01$ vs. the Con group. LASS2, LAG1 longevity assurance homolog 2; Con, control; shRNA, short hairpin RNA.

1:100, Santa Cruz Biotechnology, Inc.) was applied to each slide overnight at $4^{\circ} \mathrm{C}$. Following washing with PBS (pH 7.4), the slides were incubated for $30 \mathrm{~min}$ with a rabbit anti-mouse secondary antibody (cat. no. A-11062, dilution, 1:400; Invitrogen; Thermo Fisher Scientific, Inc.). Subsequently, the slides were stained with diaminobenzidine, counterstained with hematoxylin, dehydrated in ethanol, cleared in xylene, mounted on coverslips and examined under an optical microscope (Eclipse E200; Nikon Corporation, Tokyo, Japan). The cells with yellow-brown staining in their cytoplasm were considered LASS2-positive. The mean density of staining, calculated using Image Pro-Plus (Media Cybernetics, Inc., Rockville, MD, USA) as previously described (16), was used to quantify the LASS2 staining.

MMP zymography. To investigate MMP-2 and MMP-9 activity in the xenografts expressing different LASS2 levels, gelatin zymography was used. SDS-PAGE on a $10 \%$ gel containing $0.1 \mathrm{mg} / \mathrm{ml}$ gelatin was used for electrophoresis. The protein samples ( $20 \mu \mathrm{g}$ of each) were loaded into each lane, and electrophoresis was performed at $100 \mathrm{~V}$ for $1.5 \mathrm{~h}$ at $4^{\circ} \mathrm{C}$. Subsequently, the gel was washed twice with $100 \mathrm{ml}$ solution containing $2.5 \%$ Triton $\mathrm{X}-100$ on a rotary shaker for $40 \mathrm{~min}$ at room temperature, then incubated in $100 \mathrm{ml}$ reaction buffer $\left(50 \mathrm{mmol} / 1\right.$ Tris- $\mathrm{HCl}, 5 \mathrm{mmol} / 1 \mathrm{CaCl}_{2}, 0.02 \%$ $\mathrm{NaN}_{3}, \mathrm{pH} 7.6$ ) for $42 \mathrm{~h}$ at $37^{\circ} \mathrm{C}$. Following staining with
Coomassie brilliant blue and destaining with methanol and acetic acid, the gels were scanned using the BioSpectrum Imaging System (BioSpectrum 510; UVP, Inc., Upland, CA, USA), and the relative activities of MMP-2 and MMP-9 were quantified by densitometric analysis of the zymograms using ImageJ software (National Institutes of Health, Bethesda, MD, USA).

Statistical analysis. All data were expressed as the mean \pm standard deviation. Statistical analyses were performed using SPSS software (version 19.0; IBM SPSS, Armonk, NY, USA). A one-way analysis of variance followed by a Tukey's multiple comparison test was used to evaluate the differences between the groups. $\mathrm{P}<0.05$ was considered to indicate a statistically significant difference.

\section{Results}

Expression of LASS2 in EJ-M3 cells following transfection with a LASS2 shRNA construct or a LASS2 overexpression plasmid. To confirm transfection efficiency, fluorescence microscopy was used to detect the expression of GFP in EJ-M3 cells transfected with a LASS2 shRNA construct or a LASS2 overexpression plasmid $72 \mathrm{~h}$ following transfection (Fig. 1A). Non-transfected EJ-M3 cells expressing GFP alone were used as the Con group. Subsequently, the cells were collected to 


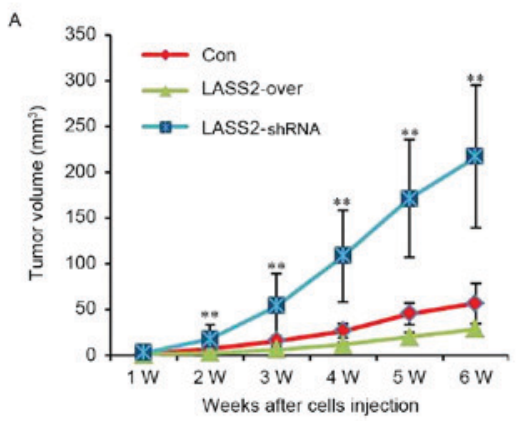

$\mathrm{C}$

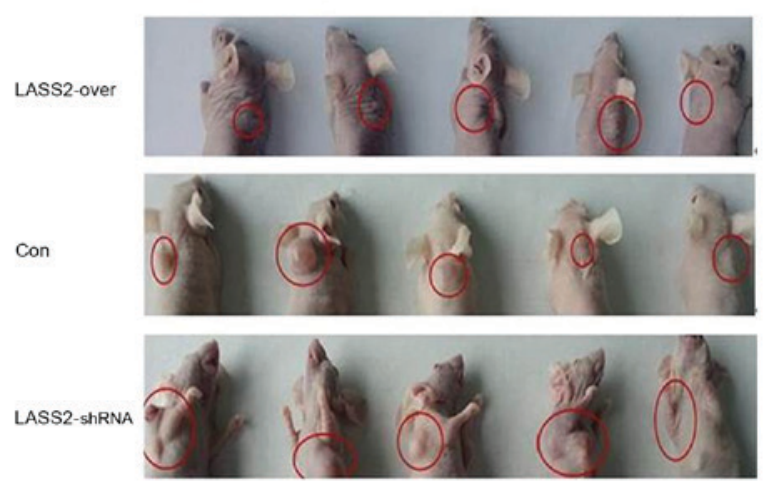

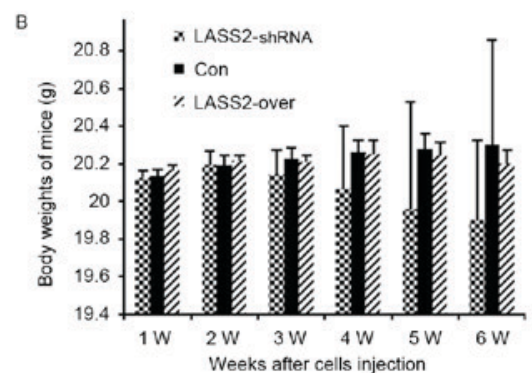

D

LASS2-over

Con

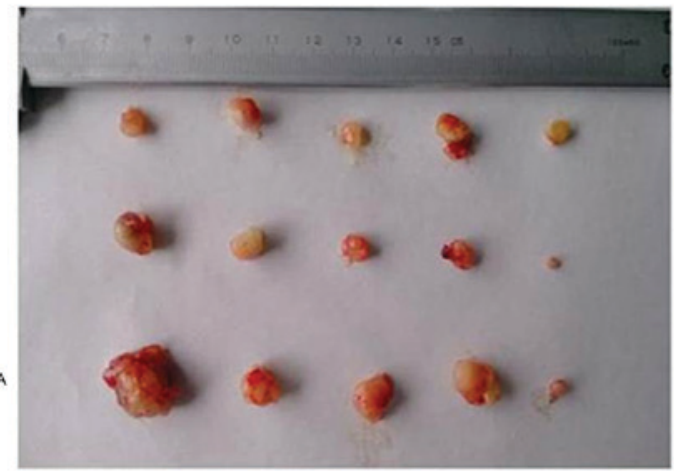

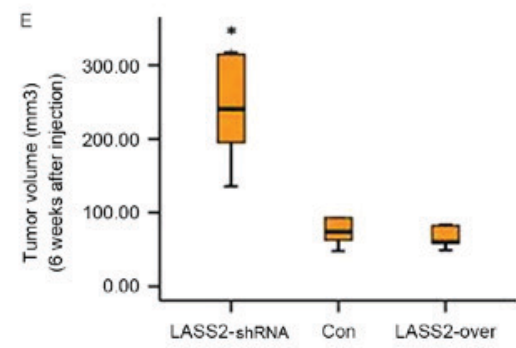

Figure 2. Knockdown or overexpression of LASS2 in EJ-M3 cells impact tumor growth in nude mice. EJ-M3 cells were transfected with a LASS2-over plasmid or a LASS2 shRNA plasmid, and then injected subcutaneously into the right dorsal aspect of the neck of nude mice. Non-infected EJ-M3 cells were used as the Con. The (A) tumor volumes and (B) body weights of mice were measured weekly until 6 weeks following injection. (C) Images of nude mice were taken 6 weeks following injection. (D) A total of 6 weeks following injection, all mice were sacrificed, and the tumors were resected and assessed. (E) Tumor volumes were measured following tumor resection. ${ }^{*} \mathrm{P}<0.05,{ }^{* *} \mathrm{P}<0.01$ vs. the Con group. LASS2, LAG1 longevity assurance homolog 2; Con, control; LASS2-over, LASS2 overexpression group; shRNA, short hairpin RNA.

detect the expression of LASS2 at the mRNA and protein levels by RT-qPCR and western blot analysis, respectively. $\beta$-Actin was used as the internal control for normalization. The RT-qPCR results demonstrated that LASS2 expression in the LASS2-shRNA group was significantly decreased by $39 \%$ $(\mathrm{P}<0.001)$, while that in the LASS2-over group was significantly increased by $263 \%$ ( $\mathrm{P}<0.001)$, compared with the Con group (Fig. 1B). A western blot analysis demonstrated similar results at the protein level (Fig. 1C and D). These results demonstrate that the two different recombinant LASS2 plasmids were successfully transfected into the EJ-M3 cells.

Knockdown or overexpression of LASS2 significantly affects bladder tumor growth in vivo. To determine the functional role of LASS2 in human bladder cancer initiation and progression, EJ-M3 cells transfected with LASS2 shRNA constructs or LASS2 overexpression plasmids were subcutaneously injected into the right side of the dorsal aspect of the neck of nude mice. Non-transfected EJ-M3 cells were injected as the Con group. Tumor growth and body weight were closely monitored during the following weeks (Fig. 2). Ten days following injection, palpable subcutaneous xenografts were observed in all nude mice, and the tumor incidence rates in the three groups were $100 \%$. Tumor growth curves demonstrated that the xenografts grew significantly faster in the LASS2-shRNA group compared with both the LASS2-over and Con groups (both $\mathrm{P}<0.001$ ); however, no significant difference was observed between the LASS2-over and Con groups ( $\mathrm{P}>0.05)$ (Fig. 2A). No animals died during the observation period. Six weeks following injection, all mice were sacrificed and the tumors were removed. All subcutaneous xenografts exhibited oval or nodal shapes with a smooth surface (Fig. 2C and D). The tumor volumes of the LASS2-shRNA, LASS2-over and Con groups were $222.32 \pm 124.97,41.01 \pm 15.97$ and $69.60 \pm 31.37 \mathrm{~mm}^{3}$, respectively. Compared with the Con group, the TIRs in the LASS2-shRNA and LASS2-over groups were -219.42 and $41.08 \%$, respectively (data not shown). These results indicate 
A

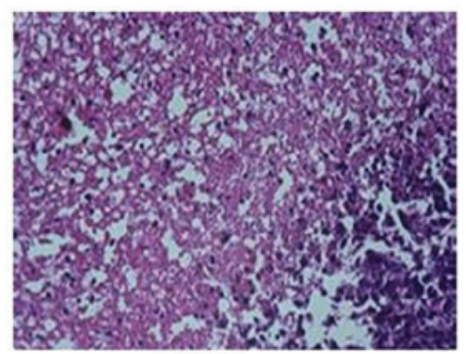

LASS2-over

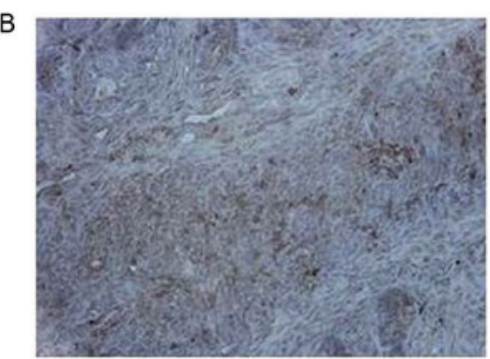

LASS2-over

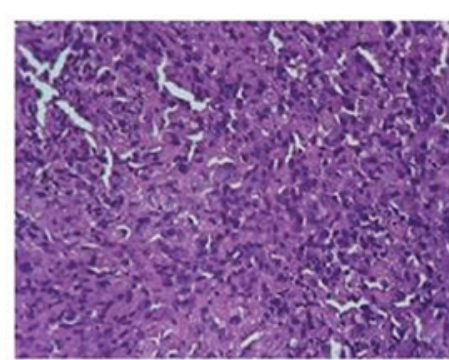

Con

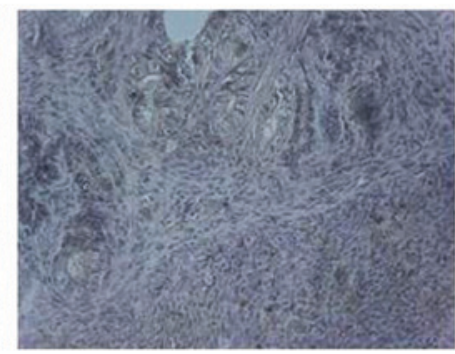

Con

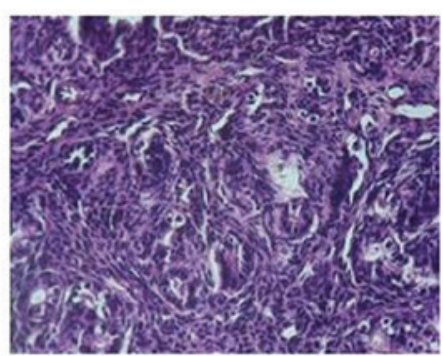

LASS2-shRNA

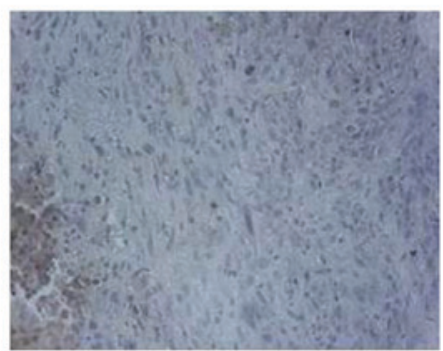

LASS2-shRNA

C

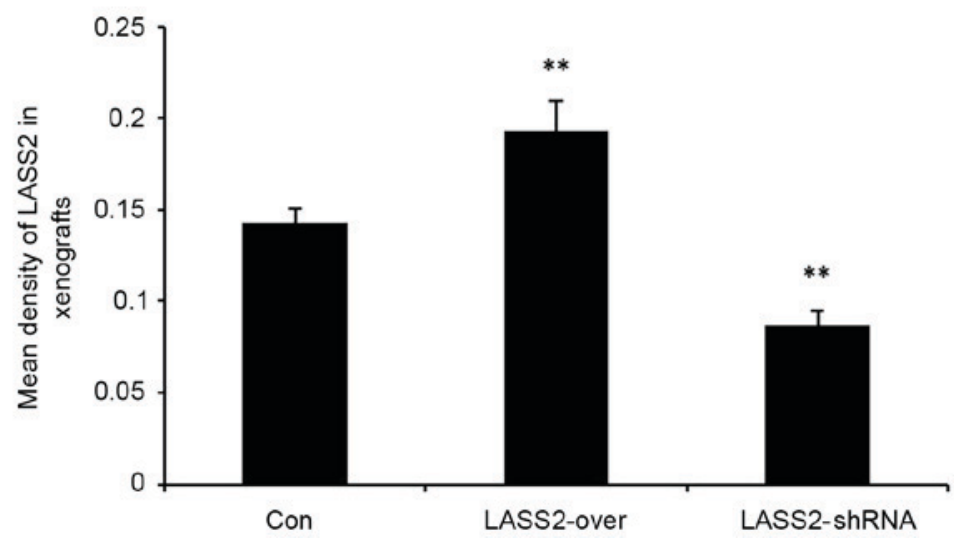

Figure 3. Histology analysis of LASS2 protein expression in xenografts from nude mice. Images of (A) hematoxylin \& eosin and (B) immunohistochemistry staining for LASS2 in the xenografts of nude mice injected with EJ-M3 cells were captured at x400 magnification. (C) Immunohistochemical staining for LASS2 was quantified as a mean density using Image Pro-Plus software. ${ }^{*} \mathrm{P}<0.05,{ }^{* *} \mathrm{P}<0.01$ vs. the Con group. LASS2, LAG1 longevity assurance homolog 2 ; Con, control; LASS2-over, LASS2 overexpression group; shRNA, short hairpin RNA.

that LASS2 knockdown significantly increases tumor volume, while LASS2 overexpression does not significantly affect tumor growth when compared with the Con group, despite a trend for LASS2 overexpression to decrease tumor volume $(\mathrm{P}=0.821$; Fig. 2E). During the experimental period, LASS2 knockdown had a tendency to reduce the body weight of nude mice; however, there was no significant difference in body weight between these three groups (Fig. 2B).

H\&E staining confirmed the subcutaneous xenografts as bladder transitional cell carcinoma. The tumor cells exhibited a nest-like distribution, apparent heteromorphism, and large and irregular nuclei with obvious nucleoli, particularly in the LASS2-shRNA group (Fig. 3A). Additionally, immunohistochemical analysis was used to detect LASS2 expression levels in the xenografts. There was a significantly higher mean density of LASS2-positive cells in the LASS2-over group compared with those in the LASS2-shRNA and Con groups $(\mathrm{P}<0.001$; Fig. $3 \mathrm{~B}$ and $\mathrm{C})$. These results suggest that different LASS2 expression levels regulate the tumorigenicity of bladder cancer EJ-M3 cells, the growth of xenografts and the degree of tumor cell heteromorphism in vivo.
Effect of LASS2 expression on MMP-2 and MMP-9 activity in xenografts. Using gelatin zymography and a quantified analytical system, MMP-2 and MMP-9 activity in the xenografts with different LASS2 expression levels was analyzed. MMP-2 and MMP-9 expression levels were identified as clear bands against a lighter blue background (Fig. 4A). Compared with the Con group, the relative activities of MMP-2 (Fig. 4B) and MMP-9 (Fig. 4C) in the LASS2-shRNA group were significantly higher $(\mathrm{P}<0.0001)$, while those in the LASS2-over group were significantly lower $(\mathrm{P}<0.0001)$.

\section{Discussion}

LASS2 serves an important role in inhibiting the proliferation and invasion of a number of tumor cancer cell lines, including those of the prostate, liver and breast $(7,17,18)$. Previous studies have suggested that LASS2 inhibits tumor invasion and metastasis through the inhibition of vacuolar-H(+)-ATPase by LASS2 through its association with the V-type proton ATPase $16 \mathrm{kDa}$ proteolipid subunit (the $\mathrm{C}$ subunit of V-ATPase) may subsequently suppress the activation of hydrogen sensitive 
A
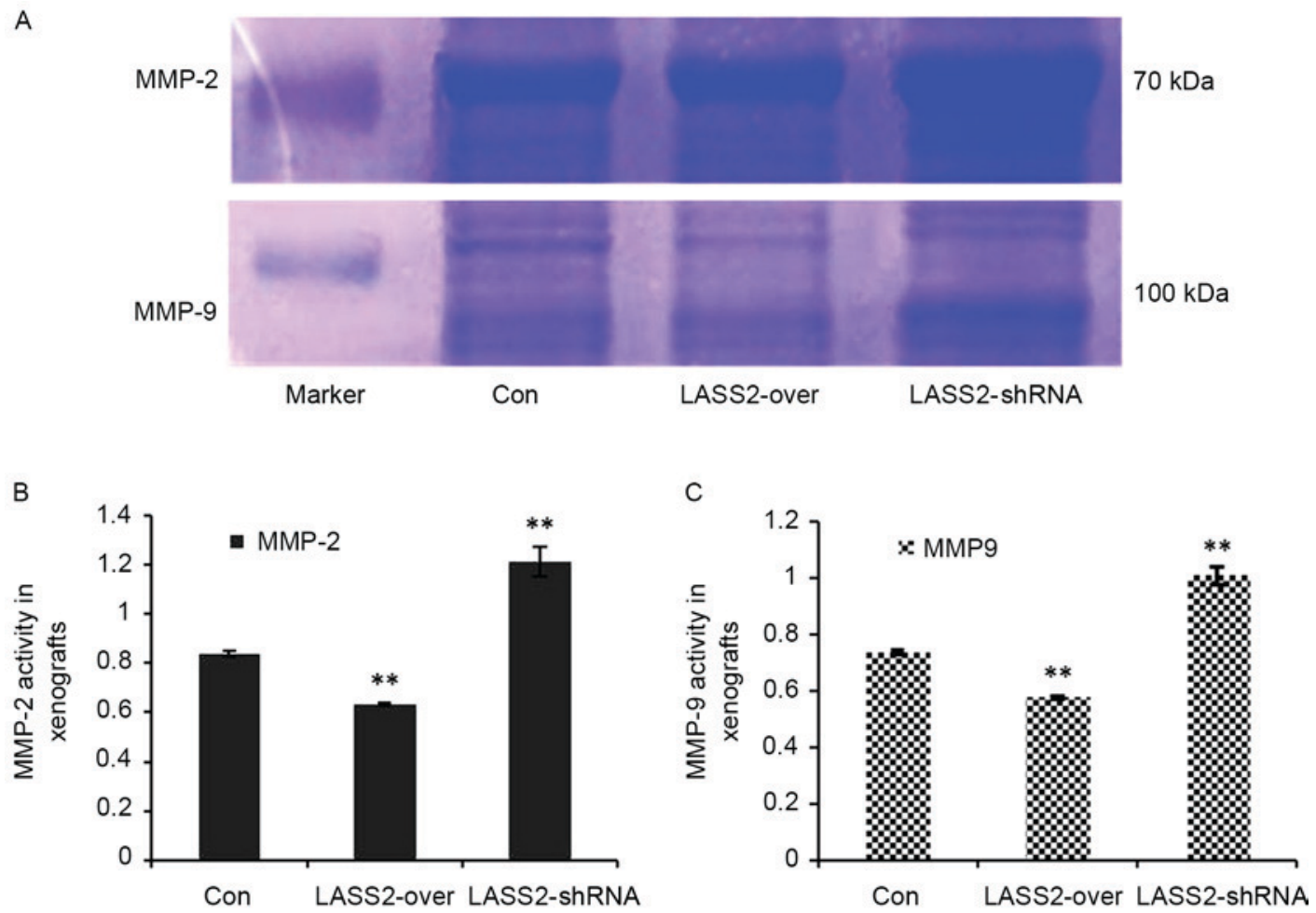

Figure 4. Gelatin zymography analysis of the activities of MMP-2 and MMP-9 in the xenografts from nude mice. (A) Representative images of MMP zymography. Bar diagrams representing the relative activity of (B) MMP-2 and (C) MMP-9. $\mathrm{P}<0.05,{ }^{* *} \mathrm{P}<0.01$ vs. the Con group. MMP, matrix metalloproteinase; LASS2, LAG1 longevity assurance homolog 2; Con, control; LASS2-over, LASS2 overexpression group; shRNA, short hairpin RNA.

proteolytic enzymes and the degradation of extracellular matrix, inducing apoptosis of tumor cells $(4-7,19,20)$. Previous studies have also revealed that V-ATPase serves a critical role in the secretion and activation of degradation enzymes, such as MMPs $(21,22)$.

LASS2 has been demonstrated to promote tumor cell apoptosis via the synthesis of ceramide $(3,23,24)$. Tang et al (7) transiently transfected HCCLM3 cells with a pCMV-HA2-LASS2 plasmid in order to increase the expression of LASS2, and the results suggested that LASS2 overexpression inhibits the migration and invasion of HCCLM3 cells. Lu et al (25) demonstrated that the risk of hepatocellular carcinoma was elevated in 1-month-old mice in which LASS2 had been knocked out. Previous studies have demonstrated that LASS2 expression is downregulated and associated with poor clinical prognosis in advanced human bladder carcinoma (26), and that LASS2 expression is significantly correlated with diverse proliferation, metastasis and invasion in bladder cancer cells (13). Another previous study revealed that LASS2 overexpression downregulated the expression of apoptosis regulator Bcl-2 and survivin, while LASS2 siRNA upregulated their expression (27). A recent study by our group demonstrated that LASS2 overexpression in the bladder cancer EJ cell line resulted in a decrease in cell proliferation, metastasis and invasion ability in vitro (28). These results are in agreement with the previous studies discussed above.

Recent studies have revealed that the silencing of LASS2 in a number of tumor cell lines increases tumor growth and lymph node metastases in vivo $(20,29)$. In the present study, the results of RT-qPCR and Western blot analyses confirmed that the EJ-M3-LASS2-knockdown cells and
EJ-M3-LASS2-overexpression cells were successfully established by transfecting LASS2-shRNA or LASS2 overexpression plasmids into the human bladder cancer EJ-M3 cell line. The cell suspensions were subcutaneously injected into the right dorsal aspect of the neck of the nude mice in order to observe xenograft tumor growth. According to the results of the tumor growth curves produced and the TIRs calculated, LASS2 knockdown in human bladder cancer EJ-M3 cells significantly promoted the growth of xenografts in nude mice. Additionally, H\&E staining and the xenograft experiments suggested that different LASS2 expression levels affect the degree of tumor cells heteromorphism.

Data from the present study demonstrated that LASS2 overexpression had a tendency to inhibit the growth of xenografts; however, this result was not statistically significant. Previous studies have demonstrated that LASS2 overexpression inhibits tumor growth in vivo; for example, Fan et al (8) demonstrated that the combination of LASS2 overexpression and doxorubicin significantly inhibited the growth of xenografts in nude mice. Additionally, several previous studies have revealed that reducing MMP-2 and MMP-9 activity inhibited tumorigenicity and the growth of xenografts in nude mice. For example, Xu et al (30) reported that the silencing of LASS2 may promote invasion of human prostate cancer cell in vitro by increasing the activity of secreted MMP-2. Consistently, Mei et al (18) reported that the overexpression of LASS2 could inhibit the invasion of the human breast cancer cell line MCF-7 in vitro by suppressing MMP-2 activation and extracellular matrix degradation. In addition, a recent study has revealed that MMP-9 knockdown inhibited tumorigenicity in nude mice (31). 
In order to further investigate the influence of LASS2 overexpression on the growth of bladder tumor xenografts, LASS2 expression and MMP-2/-9 activity in the xenografts was detected using immunohistochemistry and gelatin zymography, respectively. The activities of MMP-2 and MMP-9 were negatively correlated with LASS2 expression in the xenografts of nude mice. These results suggest that LASS2 overexpression significantly suppresses the activities of MMP-2 and MMP-9 in vivo, which is consistent with the results of previous studies $(18,20,30)$.

The data from the present study revealed that LASS2 overexpression had a tendency to inhibit the growth of bladder tumor xenografts, though this was not statistically significant. However, the activities of MMP-2 and MMP-9 were significantly decreased in LASS2 overexpression xenografts compared with the Con. Additionally, there was a wide variability in tumor volumes within the same groups. There are several possible explanations for this, such as that the mechanisms of tumorigenicity and tumor growth in vivo are complex, including gene-gene interaction and multiple factors involved in tumor formation, growth and metastasis. Another possible explanation is the short observation period and the small sample size of the present study. Therefore, further research is required to confirm whether LASS2 overexpression inhibits tumor growth.

In conclusion, the results from the present study indicate that LASS2 knockdown enhances the heteromorphism of EJ-M3 cell xenograft tumors and promotes the tumorigenicity and growth of tumors in vivo. In addition, LASS2 overexpression was identified to have a tendency to inhibit the growth of xenografts, suggesting that it is a potential therapeutic target for bladder cancer.

\section{Acknowledgements}

The present study was supported by the National Natural Science Foundation of China (grant nos. 81260374 and 81460384), the Yunnan Provincial Department of Education Fund (grant no. 2014Z072), the Joint Project of Science and Technology of Yunnan and Kunming Medical Universities (grant nos. 2014FA015 and 2014FZ031), the Yunnan Provincial Health Department Project (grant no. 2014NS081) and the Yunnan Provincial Science and Technology Project (grant no. 2015FB196).

\section{References}

1. Torre LA, Bray F, Siegel RL, Ferlay J, Lortet-Tieulent J and Jemal A: Global cancer statistics, 2012. CA Cancer J Clin 65: 87-108, 2015.

2. Siegel RL, Miller KD and Jemal A: Cancer statistics, 2017. CA Cancer J Clin 67: 7-30, 2017.

3. Ma C, Liu Y, Zheng J, Fang W, You J, Wang J, Cui X and Wu B: Identification of tumor metastasisrelated gene TMSG-1 by mRNA differential display. Sci China C Life Sci 45: 553-560, 2002.

4. Yu W, Wang L, Wang Y, Xu X, Zou P, Gong M, Zheng J, You J, Wang H, Mei F and Pei F: A novel tumor metastasis suppressor gene LASS2/TMSG1 interacts with vacuolar ATPase through its homeodomain. J Cell Biochem 114: 570-583, 2013.

5. Xu XY, You JF, Pei F and Zhang B: Silencing of tumor metastasis suppressor gene 1 promotes invasion of prostate cancer cell in vitro and its molecular mechanisms. Beijing Da Xue Xue Bao 43: 814-819, 2011 (In Chinese).
6. Xu X, You J and Pei F: Silencing of a novel tumor metastasis suppressor gene LASS2/TMSG1 promotes invasion of prostate cancer cell in vitro through increase of vacuolar ATPase activity. J Cell Biochem 113: 2356-2363, 2012.

7. Tang N, Jin J, Deng Y, Ke RH, Shen QJ, Fan SH and Qin WX: LASS2 interacts with V-ATPase and inhibits cell growth of hepatocellular carcinoma. Sheng Li Xue Bao 62: 196-202, 2010 (In Chinese)

8. Fan S, Niu Y, Tan N, Wu Z, Wang Y, You H, Ke R, Song J, Shen Q, Wang W, et al: LASS2 enhances chemosensitivity of breast cancer by counteracting acidic tumor microenvironment through inhibiting activity of V-ATPase proton pump. Oncogene 32: $1682-1690,2013$

9. Schiffmann S, Sandner J, Birod K, Wobst I, Angioni C, Ruckhäberle E, Kaufmann M, Ackermann H, Lötsch J, Schmidt H, et al: Ceramide synthases and ceramide levels are increased in breast cancer tissue. Carcinogenesis 30: 745-752, 2009.

10. Wang H, Wang J, Zuo Y, Ding M, Yan R, Yang D and Ke C: Expression and prognostic significance of a new tumor metastasis suppressor gene LASS2 in human bladder carcinoma. Med Oncol 29: 1921-1927, 2012.

11. Yang D, Wang H, Wang J, Zhang $\mathrm{C}$ and $\mathrm{Xu} \mathrm{H}$ : Establishment of a fluorescent implantation metastasis model of bladder cancer and real-time microscopic detection in nude mice. Asian Pacific J Cancer Prev 12: 393-396, 2011.

12. Girnita A, All-Ericsson C, Economou MA, Aström K, Axelson M, Seregard S, Larsson O and Girnita L: The insulin-like growth factor-I receptor inhibitor picropodophyllin causes tumor regression and attenuates mechanisms involved in invasion of uveal melanoma cells. Clin Cancer Res 12: 1383-1391, 2006.

13. Zhao Q, Wang H, Yang M, Yang D, Zuo Y and Wang J: Expression of a tumor-associated gene, LASS2, in the human bladder carcinoma cell lines BIU-87, T24, EJ and EJ-M3. Exp Ther Med 5: 942-946, 2013.

14. Livak KJ and Schmittgen TD: Analysis of relative gene expression data using real-time quantitative PCR and the 2(-Delta Delta C(T)) method. Methods 25: 402-408, 2001.

15. Cumashi A, Tinari N, Rossi C, Lattanzio R, Natoli C, Piantelli M and Iacobelli S: Sunitinib malate (SU-11248) alone or in combination with low-dose docetaxel inhibits the growth of DU-145 prostate cancer xenografts. Cancer Lett 270: 229-233, 2008

16. Wang CJ, Zhou ZG, Holmqvist A, Zhang H, Li Y, Adell G and Sun XF: Survivin expression quantified by image Pro-Plus compared with visual assessment. Appl Immunohistochem Mol Morphol 17: 530-535, 2009.

17. $\mathrm{Su}$ J, You JF, Wang JL, Cui XL, Fang WG and Zheng J: Overexpression of human tumor metastasis-related gene TMSG-1 suppresses cell proliferation and invasion of a highly metastatic prostate cancer cell line PC-3M-1E8 in vitro. Zhonghua Zhong Liu Za Zhi 30: 404-407, 2008 (In Chinese).

18. Mei F, You J, Liu B, Zhang M, Liu J, Zhang B and Pei F: LASS2/TMSG1 inhibits growth and invasion of breast cancer cell in vitro through regulation of vacuolar ATPase activity. Tumour Biol 36: 2831-2844, 2015.

19. Xu XY, Pei F and You JF: TMSG-1 and its roles in tumor biology. Chin J Cancer 29: 697-702, 2010

20. Xu X, Liu B, Zou P, Zhang Y, You J and Pei F: Silencing of LASS2/TMSG1 enhances invasion and metastasis capacity of prostate cancer cell. J Cell Biochem 115: 731-743, 2014.

21. Sennoune SR, Luo D and Martinez-Zaguilán R: Plasmalemmal vacuolar-type H+-ATPase in cancer biology. Cell Biochem Biophys 40: 185-206, 2004.

22. Fais S, De Milito A, You H and Qin W: Targeting vacuolar $\mathrm{H}+-\mathrm{ATPases}$ as a new strategy against cancer. Cancer Res 67: 10627-10630, 2007.

23. Rossi MJ, Sundararaj K, Koybasi S, Phillips MS, Szulc ZM, Bielawska A, Day TA, Obeid LM, Hannun YA and Ogretmen B: Inhibition of growth and telomerase activity by novel cationic ceramide analogs with high solubility in human head and neck squamous cell carcinoma cells. Otolaryngol Head Neck Surg 132: 55-62, 2005.

24. Su J, Yu W, Gong M, You J, Liu J and Zheng J: Overexpression of a novel tumor metastasis suppressor gene TMSG1/LASS2 induces apoptosis via a caspase-dependent mitochondrial pathway. J Cell Biochem 116: 1310-1317, 2015.

25. Lu X, Chen Y, Zeng T, Chen L, Shao Q and Qin W: Knockout of the HCC suppressor gene Lass2 downregulates the expression level of miR-694. Oncol Rep 32: 2696-2702, 2014. 
26. Wang H, Wang J, Zuo Y, Ding M, Yan R, Yang D and Ke C: Expression and prognostic significance of a new tumor metastasis suppressor gene LASS2 in human bladder carcinoma. Med Oncol 29: 1921-1927, 2012

27. Wang H, Zhang W, Zuo Y, Ding M, Ke C, Yan R, Zhan H, Liu J and Wang J: miR-9 promotes cell proliferation and inhibits apoptosis by targeting LASS2 in bladder cancer. Tumour Biol 36: 9631-9640, 2015.

28. Wang H, Zuo Y, Ding M, Ke C, Yan R, Zhan H, Liu J, Wang W, Li N and Wang J: LASS2 inhibits growth and invasion of bladder cancer by regulating ATPase activity. Oncol Lett 13: 661-668, 2017.

29. Chen L, Lu X, Zeng T, Chen Y, Chen Q, Wu W, Yan X, Cai H, Zhang Z, Shao Q and Qin W: Enhancement of DEN-induced liver tumourigenesis in hepatocyte-specific Lass2-knockout mice coincident with upregulation of the TGF- $\beta 1$-Smad4-PAI- 1 axis. Oncol Rep 31: 885-893, 2014.
30. Xu X, You J and Pei F: Silencing of a novel tumor metastasis suppressor gene LASS2/TMSG1 promotes invasion of prostate cancer cell in vitro through increase of vacuolar ATPase activity. J Cell Biochem 113: 2356-2363, 2012.

31. Guo F, Tian J, Cui M, Fang M and Yang L: Downregulation of matrix metalloproteinase 9 by small interfering RNA inhibits the tumor growth of ovarian epithelial carcinoma in vitro and in vivo. Mol Med Rep 12: 753-759, 2015. 